\title{
Vibration-induced elastic deformation of Fabry-Perot cavities
}

\author{
Lisheng Chen,* John L. Hall, and Jun Ye ${ }^{\dagger}$ \\ JILA, National Institute of Standards and Technology and University of Colorado, Boulder, Colorado 80309-0440, USA
}

Tao Yang, Erjun Zang, and Tianchu Li

Quantum Division, National Institute of Metrology, Beijing, 10013, China

(Received 29 June 2006; published 2 November 2006)

\begin{abstract}
We perform a detailed numerical analysis of Fabry-Perot cavities used for state-of-the-art laser stabilization. Elastic deformation of Fabry-Perot cavities with various shapes and mounting methods is quantitatively analyzed using finite-element analysis. We show that with a suitable choice of mounting schemes it is feasible to minimize the susceptibility of the resonator length to vibrational perturbations. This investigation offers detailed information on stable optical cavities that may benefit the development of ultrastable optical local oscillators in optical atomic clocks and precision measurements probing the fundamental laws of physics.
\end{abstract}

DOI: 10.1103/PhysRevA.74.053801

\section{INTRODUCTION}

Laser radiation with high spectral purity and long-term stability has important applications in many fields such as high-precision laser spectroscopy, optical frequency metrology, and test of fundamental physical postulates. Highfinesse Fabry-Perot cavities are widely used in frequency stabilization of various laser systems. In this task feedback control is employed to guide the laser frequency to be within a small fraction of the linewidth of one of the cavity resonances, eliminating the intrinsic noises of the laser and replacing them with the measurement noise associated with the Fabry-Perot cavity resonance. For a cavity with a moderately high finesse of $\sim 50000$, a resonance linewidth of $30 \mathrm{kHz}$ is readily obtained. This frequency resonance can have a large contrast from the shot-noise-limited background and is not power saturated, features that are particularly useful in obtaining a high signal-to-noise ratio $(S / N)$. Additionally, when the reflection field of the cavity is employed for error signal generations, the frequency control bandwidth is not limited by the cavity linewidth [1]. The possibility of achieving laser radiations with a long coherence time was investigated [2]. This line of research has made steady progress [3-7] and has continuously upgraded the resolving power of spectroscopic features [8-15].

In particular, stable laser local oscillators serve as an indispensable ingredient in optical clocks that have anticipated frequency accuracy reaching $10^{-18}[11,16,17]$. In these optical clocks with resonance quality factors $(Q)>10^{14}$, Doppler broadening and collisional frequency shift can be suppressed by confining single ion $[11,18]$ or neutral atoms $[15,17,19,20]$ in the Lamb-Dicke regime [21]. On the other hand, "optical flywheels" based on femtosecond modelocked lasers provide the clockwork and establish a phasecoherent link with the rf frequency standard [22-27]. Parallel

\footnotetext{
*Wuhan Institute of Physics and Mathematics, Chinese Academy of Sciences, Wuhan, 430071, China. Electronic address: lchen@wipm.ac.cn

†Electronic address: Ye@jila.colorado.edu
}

to these encouraging breakthroughs is the development of an ultrastable probe laser capable of interrogating clock transitions in ions and neutral atoms. Induced by hyperfine coupling or external fields, these nearly forbidden clock transitions can preserve a long coherence time of $1-1000 \mathrm{~s}$ [28-31]. To obtain the line-center information with projected accuracy, the frequency characteristics of the probe laser should be compatible with the extremely narrow linewidth associated with the clock transition. Toward this goal, it is vital to have in-depth studies of major problems $[4,32]$ that affect the short- and long-term stabilities of passive FabryPerot cavities used for laser frequency stabilization. Environmental vibration is one of those dominant noise sources.

Vibration perturbations degrade the stability of the optical length between two mirrors. The structural support transmits seismic vibrations to the cavity spacer. If not properly isolated, airborne sound pressures act directly on the cavity. These perturbations induce elastic deformation along the instantaneous direction of the random force, and the effect is coupled to other directions through a nonzero Poisson ratio. Length fluctuation then acts as a broadband noise source whose Fourier spectrum modulates the laser carrier frequency, resulting in a linewidth broadening. While the airborne acoustic perturbations can be attenuated effectively by evacuating the chamber housing the cavity, coping with seismic vibrations poses technical challenges. With elaborate vibration isolation, the Bergquist group at NIST realized a subhertz laser system $\left(\sim 10^{15} \mathrm{~Hz}\right)$ that is referenced to FabryPerot cavities [4]. Nd:YAG and diode lasers stabilized to cavities with passive or active vibration isolation have been demonstrated $[5,7,33]$. Currently, passive or active isolation systems can achieve vibration attenuations at Fourier frequencies $\gtrsim 1 \mathrm{~Hz}$. It becomes more difficult and costly to construct isolation systems with lower natural frequencies while maintaining sufficient damping at these frequencies where troublesome resonances can arise.

An alternative way of robust vibration resistance has also been pursued [34]. By supporting the cavity vertically near its midplane, a compact laser system with subhertz linewidth has been experimentally demonstrated $[6,15]$. Rather than focusing on the vibration attenuation, one installs the cavity 
in a particular configuration such that its critical dimension is insensitive to the vibration-induced deformation, thus alleviating the technical difficulty and cost inefficiency frequently related to the vibration attenuation. We note that the Bergquist group [35] and the PTB group [7] have also been pursuing mounting strategies to minimize the vibration sensitivity of Fabry-Perot cavities. In addition, possible mounting methods have also been considered in Ref. [36]. These ideas have stimulated interest in searching for other cavity configurations that can relax the stringent demands for vibration attenuation.

In this paper, we explore various shapes and structural supports of Fabry-Perot cavities, searching for designs that reduce the sensitivity of the cavity length to the environmental vibration perturbations. A quantitative analysis of the elastic deformation of Fabry-Perot cavities provides valuable guidance for cavity design. To our knowledge, however, a comprehensive investigation of the elastic deformation of Fabry-Perot cavities at the modern precision level is not available in the literature. Here we use finite-element analysis (FEA) to perform a detailed numerical analysis of various Fabry-Perot cavity configurations and to identify optimal designs with improved vibration resistance. We show that such an optimization is possible for cavities mounted vertically or horizontally. In addition, a compounded cavity horizontally mounted is proposed for a test of the Lorentz invariance.

To achieve the ultimate performance of a Fabry-Perot cavity, considerable efforts have been taken to address a variety of stability issues [4,37]. Notably, Numata et al. [38] suggested that the length stability of present state-of-the-art optical cavities begins to suffer from the thermal noise in the cavity spacer and mirror substrates, as well as in the mirror coating layers. The impact of the thermal noise on the cavity performance has been evaluated [38] and experimentally confirmed [39]. Cavity materials with low mechanical loss and a proper design of low-loss mirror coatings help to reduce this noise. In addition, cryogenic cooling of the cavity can reduce the thermal noise at the square root of the temperature, but with added technical complexities. Constrained by currently available mirror coatings and materials for cavity and mirror substrate, optimizing the cavity geometry becomes a trade-off between vibration sensitivity and other physical effects that favor a longer cavity. Indeed, a shorter cavity would be better in terms of vibration resistance. However, frequency fluctuations arising from the birefringence of mirror coatings and cavity thermal noise become proportionally larger as the cavity length decreases. Besides, a longer cavity has a smaller mode spacing and hence exhibits narrower resonance linewidth for a given cavity finesse. A proper design of cavity geometry and support is thus important to allow achieving the required vibration immunity while enjoying the benefits of a relatively long cavity.

This paper is organized as follows. First we introduce the static analysis used in the numerical modeling, explaining that the dynamic problem of the vibration perturbations can be reduced to a static analysis (Sec. II). We then present the vibration sensitivities for cavities of various shapes and mounting methods (Sec. III). First, horizontally mounted cylindrical cavities are discussed (Sec. III A). Second, a vertically supported tapered cavity is analyzed in detail (Sec.



(a)

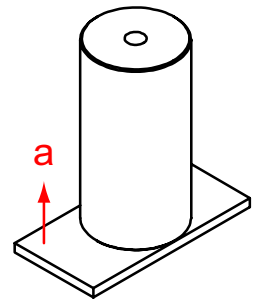

(b)
FIG. 1. (Color online) Static analysis of cavity deformation. (a) A cavity spacer vertically supported on one of its ends, with a force of gravity acting on it. The structural support is fixed (indicated as lines with multiple arrows). (b) At low frequencies, the vibrationinduced cavity deformation can be analyzed with the cavity and the structural support moving at a constant acceleration.

III B). We then return to the horizontal configuration, showing that a reduced vibration sensitivity can also be achieved for the cavities horizontally mounted (Sec. III C). As an indispensable step toward the experimental realization of various mounting schemes, we also inspect the accuracy of the numerical modeling and the stability of these cavity designs (Sec. IV). A summary and conclusion are provided at the end.

\section{STATIC ANALYSIS OF VIBRATION-INDUCED DEFORMATION}

In this section we first give a simple calculation of the elastic deformation of a cylindrical cavity, which serves as a background for our analysis of the cavity deformation. We then introduce the static analysis used in our numerical modeling. In addition, terminologies pertinent to the numerical calculation and the accompanying discussion are defined and explained in this section.

\section{A. Elastic deformation}

Figure 1(a) shows a cylindrical cavity with length $L$ standing on one of its ends, compressed vertically by gravity. For the moment we neglect the coupling among different directions due to the nonzero Poisson ratio. The fractional length change of the cavity is

$$
\Delta L / L=-\rho g L / 2 E,
$$

where $g$ is the gravitational acceleration, and $\rho$ and $E$ are the mass density and the elastic modulus of the cavity material, respectively. For a $10-\mathrm{cm}$ cavity made from ULE, the change in the optical length due to gravity is $1.6 \times 10^{-8}$ (fractional), resulting in a frequency change of $\sim 10 \mathrm{MHz} / \mathrm{g}$ at $532 \mathrm{~nm}$. Clearly, a shorter cavity will be more insensitive to vibration perturbations.

Generally the time-dependent vibration perturbation consists of a broad spectrum, and only a partial surface area of the cavity spacer is attached to its structural support. Under such circumstances, one intuitively expects the need of a full dynamic analysis of the cavity deformation. However, an approximation can be made to greatly simplify the analysis: 


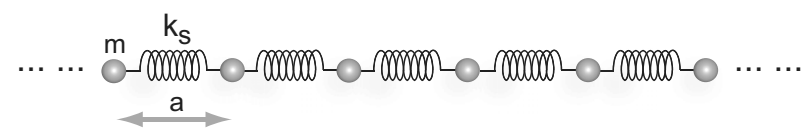

FIG. 2. One-dimensional chain of simple harmonic oscillators. At low frequencies, all point particles oscillate nearly in phase and all springs are equally compressed or stretched. $m$ and $k_{s}$ are the mass and spring constant, respectively.

At low vibration frequencies, the elastic deformation can be analyzed with the cavity and the structural support moving at a constant acceleration $a$, as shown in Fig. 1(b). Furthermore, this static acceleration can be replaced by a gravitylike force $(-m a)$ acting on the cavity and the structural support is then fixed. Thus the dynamic problem is reduced to a static analysis. The validity of this static analysis is discussed in the following section.

\section{B. Static analysis}

Excited by the vibrating support, sound waves with various frequencies propagate into all directions inside the cavity. Those with low frequencies can have wavelengths much longer than the dimension of the cavity, meaning that all particles move in phase inside each eigenmode. Consequently, the instantaneous strain (the compression or stretch of a unit volume per unit length) distributions inside the cavity closely resemble those resulting from a static force that mimics the random acceleration frozen at that moment. To see this more clearly, consider an elastic bar that deforms only along its axis. The microscopic model of the elastic bar can be simplified as a one-dimensional (1D) chain of simple harmonic oscillators (SHO's), as shown in Fig. 2. At low frequencies, all point particles in the SHO chain oscillate nearly in phase when a driving force is applied to one of the particles. As a result, all springs are equally compressed or stretched at each moment and they contribute equally to the total length change. The characteristic frequency below which the static analysis is sufficient can be estimated using the dispersion relation for an infinitely long SHO chain. This dispersion relation connects the oscillating frequency of each individual particle with the wavelength describing the collective movement of all particles in the SHO chain:

$$
\omega^{2}=\frac{4 k_{s}}{m} \sin ^{2}\left(\frac{1}{2} k a\right),
$$

where $m, k_{s}$, and $k=2 \pi / \lambda$ are the mass, spring constant, and propagation constant, respectively. Taking the continuum limit gives the familiar dispersion relation for a solid material:

$$
\omega=\sqrt{\frac{E}{\rho}} k .
$$

Using ULE as an example, Eq. (3) indicates that for $f$ $=\omega / 2 \pi<35 \mathrm{kHz}, \lambda>10 L$, where $L$ is the dimension of the material in the propagation direction of the sound wave. This derivation only gives an order-of-magnitude estimate of the characteristic frequency whose exact value depends on the

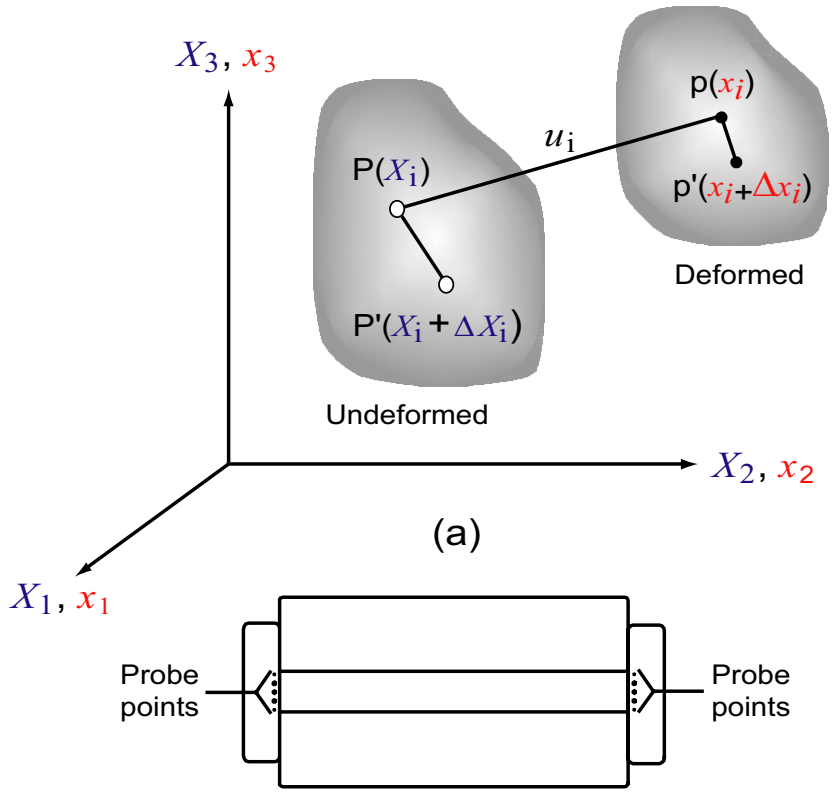

(b)

FIG. 3. (Color online) Strain and displacement in FEA. (a) Under the influence of an external force, an object is both deformed and displaced. The strain measures the fractional length change of an infinitesimal distance $P P^{\prime}$. The displacement of a point $P$ is defined as $u_{i}=x_{i}-X_{i}$. (b) The probe points (solid dots) on the cavity mirrors in an axial cross section. On each mirror the probe points span the whole diameter of the center optical hole of the cavity spacer and are evenly distributed.

details of the cavity geometry and couplings among various directions. However, in the analysis of the vibration sensitivity only low-Fourier-frequency components $(\$ 100 \mathrm{~Hz})$ are of interest because higher-frequency components can be effectively attenuated by traditional vibration isolation. At these low frequencies the static analysis is a reliable substitute for the full dynamic analysis.

\section{Strain, displacement, and constraint in FEA}

Once the dynamic problem is reduced to a static analysis, we use FEA to quantitatively investigate the elastic deformation of the Fabry-Perot cavity. Here we introduce the terminology used in FEA and in our discussions of numerical results. Figure 3(a) sketches the deformation and displacement of an object under the influence of some external force. $P$ and $p$ denote the locations of a point before and after the deformation, respectively. Along axis $i$, the displacement of a point $P$ is defined as

$$
u_{i}(X)=x_{i}-X_{i}
$$

where $x(X)$ denotes the coordinates of a point on the object with (without) the deformation. To detect the displacements of the mirror surfaces in an axial cross section, we place two sets of probe points on the two mirrors, as indicated by solid dots in Fig. 3(b). The change of optical length between two mirrors can be determined from the differential displacement of these two sets of probe points. 

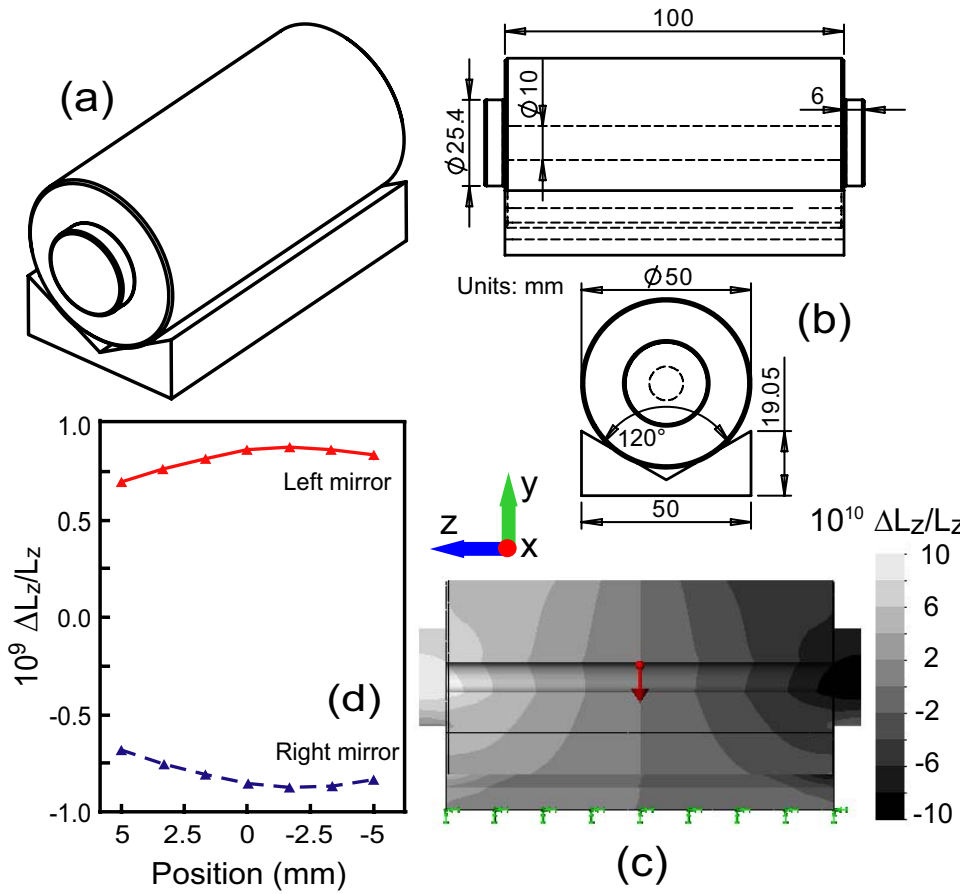

FIG. 4. (Color online) Cavity on V block. (a) Three-dimensional view of the cavity and its support. (b) Dimension of the cavity. (c) Distribution of the displacement along the $z$ axis in an axial cross section. (d) Displacements at various transverse positions (along the $y$ axis) on the reflecting surface of the mirror. The origin of the horizontal axis denotes the center of the mirror. The fractional length increase is $1.8 \times 10^{-9} / \mathrm{g}$, corresponding to a frequency excursion of $\sim 1 \mathrm{MHz} / \mathrm{g}$ at $532 \mathrm{~nm}$.
The numerical calculations are performed with FEA packages COSMOSWORKS and ANSYS. The material of the cavity is chosen as ULE (mass density, $2.21 \times 10^{3} \mathrm{~kg} / \mathrm{m}^{3}$; elastic modulus, $6.67 \times 10^{10} \mathrm{~N} / \mathrm{m}^{2}$; Poisson's ratio, 0.17). In the calculation we apply a gravitylike force on the cavity, with directions either in the vertical or in the horizontal plane. Although the magnitude of the acceleration can be arbitrarily chosen because the deformation is linearly scaled with it, we use $g=9.8 \mathrm{~m} / \mathrm{s}^{2}$ throughout the paper for the convenience of making direct comparisons among various configurations. In these analyses, either the structural support or the supporting surface of the cavity is restrained in a certain manner to prevent the mechanical structure from translational and rotational movements. Different mesh sizes are used in the numerical modeling to examine potential errors introduced by the finite mesh size.

\section{RESULTS FOR VARIOUS CAVITY SHAPES AND MOUNTING METHODS}

This section presents numerical results for cavities of various shapes and mounting methods. The discussions are organized into three parts. First we investigate cavities horizontally supported, a configuration that is widely used. We then perform detailed calculations for a tapered cavity vertically supported. The change in the cavity length is investigated with a force of gravity applied either in the vertical or in the horizontal directions. For the vertically supported tapered cavities, effects such as different taper angles are also discussed. In the third part, we return to cavities horizontally mounted, showing that with properly designed structural support, it is feasible to achieve a similar level of vibration insensitivity observed in the vertical mounting method.

\section{A. Horizontal mounting}

Horizontal mounting is a common experimental configuration in laser frequency stabilization. Typically, a cylindrical cavity is supported by a $\mathrm{V}$ block or by two U-shaped brackets. We explore these two cases by evaluating the mirror displacement resulting from the elastic deformation of the cavity. A similar analysis is also applied to a cavity with a rectangular cross section. An example of cavity design for testing Lorentz invariance is given at the end of this section.

\section{Cylindrical cavity on a V block}

Figure 4 shows a cylindrical cavity supported by a $\mathrm{V}$-shaped block and the numerical results obtained with FEA. Indicated in the figure, a force of gravity is applied to the cavity in the vertical direction and the bottom plane of the $\mathrm{V}$ block is fixed. Figures 4(a) and 4(b) give the 3D view and the dimension of the cavity, respectively. Figure 4(c) plots the distribution of the displacements along the optical (z) axis in an axial cross section. The elastic displacement shown in Fig. 4(c) reveals that the two mirrors moves apart from each other when the gravity is applied.

To evaluate the change of the optical length between the two mirrors, we place probe points on the reflecting surface of the mirror, as shown in Fig. 3(b). On each mirror the probe points span the whole diameter of the center hole and are evenly distributed. Figure 4(d) plots the $z$-direction displacements of the two mirrors sampled by the probe points along the $y$ direction. Not surprisingly, the distance between the two mirrors increases because the cavity is compressed vertically and hence it bulges in the horizontal directions. The fractional length increase is $1.8 \times 10^{-9} / \mathrm{g}$, corresponding to a frequency excursion of $\sim 1 \mathrm{MHz} / \mathrm{g}$ at $532 \mathrm{~nm}$. Also note that the two mirrors are tilted slightly from the $y$ axis because the lower portion of the cavity bears more weight load.

\section{Cylindrical cavity on two U-shaped brackets}

In the preceding example the bending of the cavity spacer is restricted by the structural support that extends the whole 


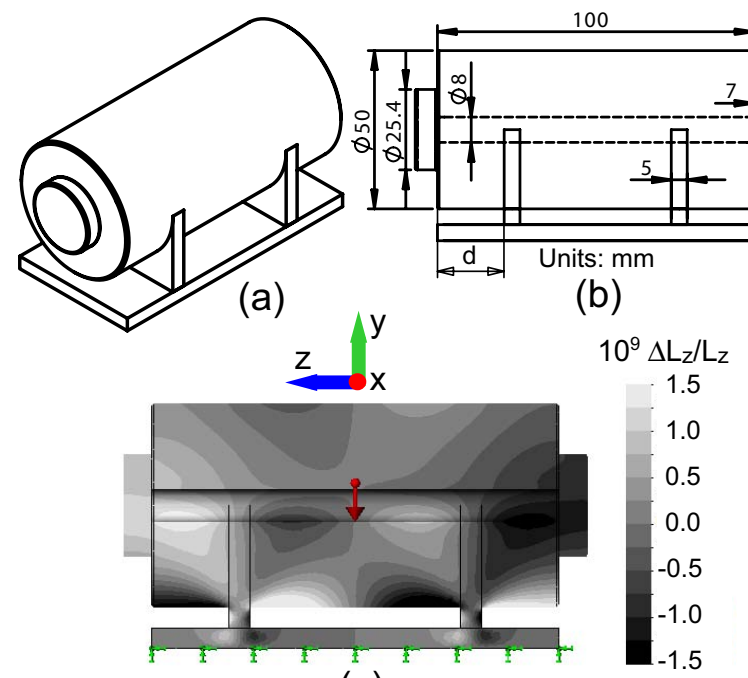

(c)

FIG. 5. (Color online) Cavity on U-shaped brackets. A force of gravity is applied to the cavity in the vertical direction and the bottom planes of the brackets are fixed in the numerical modeling. (a) Three-dimensional view of the cavity and its support. (b) Dimension of the cavity. (c) Distribution of the displacement along the $z$ direction in an axial cross section.

length of the cavity. Here we relax this restriction to investigate the effect of cavity bending on the mirror displacement. Figure 5 shows a cylindrical cavity supported by two $\mathrm{U}$-shaped brackets. Figures 5(a) and 5(b) give the 3D view and the dimension of the cavity, respectively. A force of gravity is applied to the cavity in the vertical direction, and the bottom planes of the brackets are fixed. Figure 5(c) plots the distribution of the displacement along the optical $(z)$ axis in an axial cross section. The elastic displacement shown in Fig. 5(c) reveals that, for the current positions of the two brackets shown in Fig. 5(b), the two mirrors move apart from each other and are tilted due to gravity. Definitely the mirror displacement depends on the positions of two brackets.

Mirror displacements are calculated with different values of $d$ indicated in Fig. 5(b). Figure 6 plots three typical results with $d=5,15.2$, and $21.1 \mathrm{~mm}$. Figure 6(a) represents the situation in which the supporting positions are close to the two ends of the cavity spacer. In this case, the central portion of the cavity spacer is bent downward because of gravity and the two ends are warped upward, resulting in a relatively large mirror tilt. When two supporting brackets are moved toward the middle, the weight load is redistributed in the cavity spacer. As a result, the bending around the middle of the spacer is alleviated and hence the mirror is less tilted, a situation that is quantitatively illustrated in Fig. 6(b). Inward further, there exists a balanced position at which the two mirrors are parallel to each other, independent of the vertical accelerations. The mirror displacement at this balance pivot, known as the Airy point, are shown in Fig. 6(c). As the two brackets pass the Airy point and move further toward each other, the two mirrors incline to the opposite direction, with increasing tilting angles. When supported at the Airy point [Fig. 6(c)], the fractional length increase is $2 \times 10^{-9} / \mathrm{g}$ $(1 \mathrm{MHz} / \mathrm{g}$ at $532 \mathrm{~nm})$, which is very close to the vibration sensitivity of the $\mathrm{V}$-block mounting.

The two mounting configurations discussed here concern cavities supported horizontally from the bottom. In this horizontal mounting configuration the vertical compression of the cavity induced by the gravitational pull is coupled to horizontal directions, leading to horizontal expansion and hence elongation of the optical length. In the case of U-bracket mounting the tilt of the two mirrors also modifies the optical length, as inferred from Fig. 6. Nevertheless, the gravity-induced cavity elongation still remains a performance limitation even when the cavity is supported at the Airy point. The effect of mirror tilt is negligible for a cavity directly resting on a $\mathrm{V}$-shaped block because the bending of the spacer is drastically reduced. One solution not plagued by the horizontal coupling effect is to align the optical axis along the direction of gravity and to reshape the gravityinduced cavity deformation. This vertical mounting configuration is discussed in the following section.

\section{B. Vertical mounting}

We showed in Sec. II A that a cavity vertically supported on one of its ends is compressed due to gravity. Now consider a cavity vertically supported at its midplane. Qualitatively, both the top half and the bottom half of the cavity move downward by the same amount because of the gravity, leading to a cavity length that is insensitive to vertical accelerations. To quantitatively demonstrate this cancellation ef-

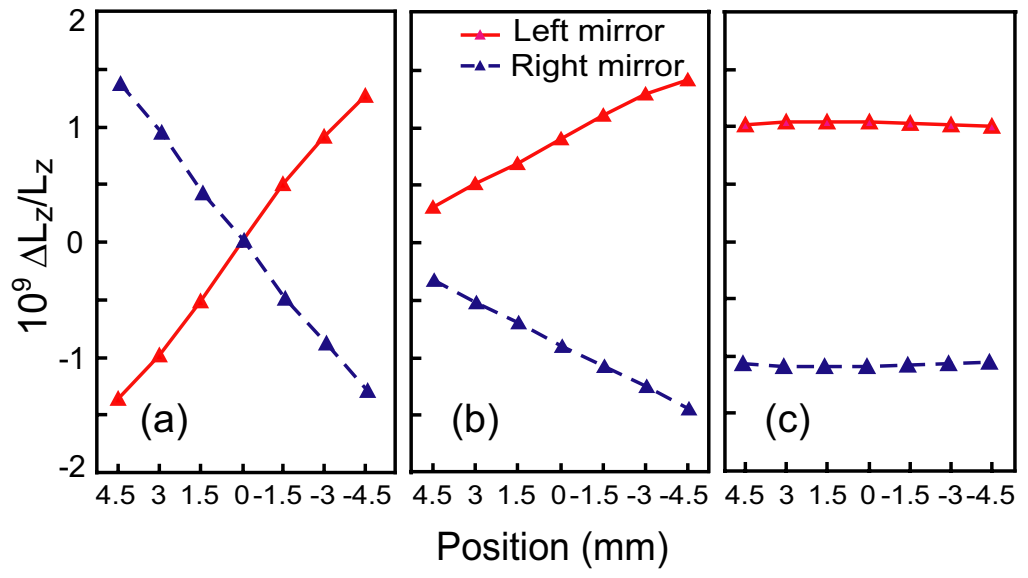

FIG. 6. (Color online) Mirror displacements of a cylindrical cavity supported horizontally by two $U$-shaped brackets. The horizontal scale represents the transverse position (along the $y$ axis) on the reflecting surface of the mirror, with the origin denoting the center of the mirror. See Fig. 5 for coordinate system. (a), (b), and (c) correspond to $d=5,15.2$, and $21.1 \mathrm{~mm}$, respectively. When the cavity is supported at the Airy point, as shown in (c), the two mirrors are parallel to each other and the corresponding fractional length increase is $2 \times 10^{-9} / \mathrm{g}(1 \mathrm{MHz} / \mathrm{g}$ at $532 \mathrm{~nm})$. 

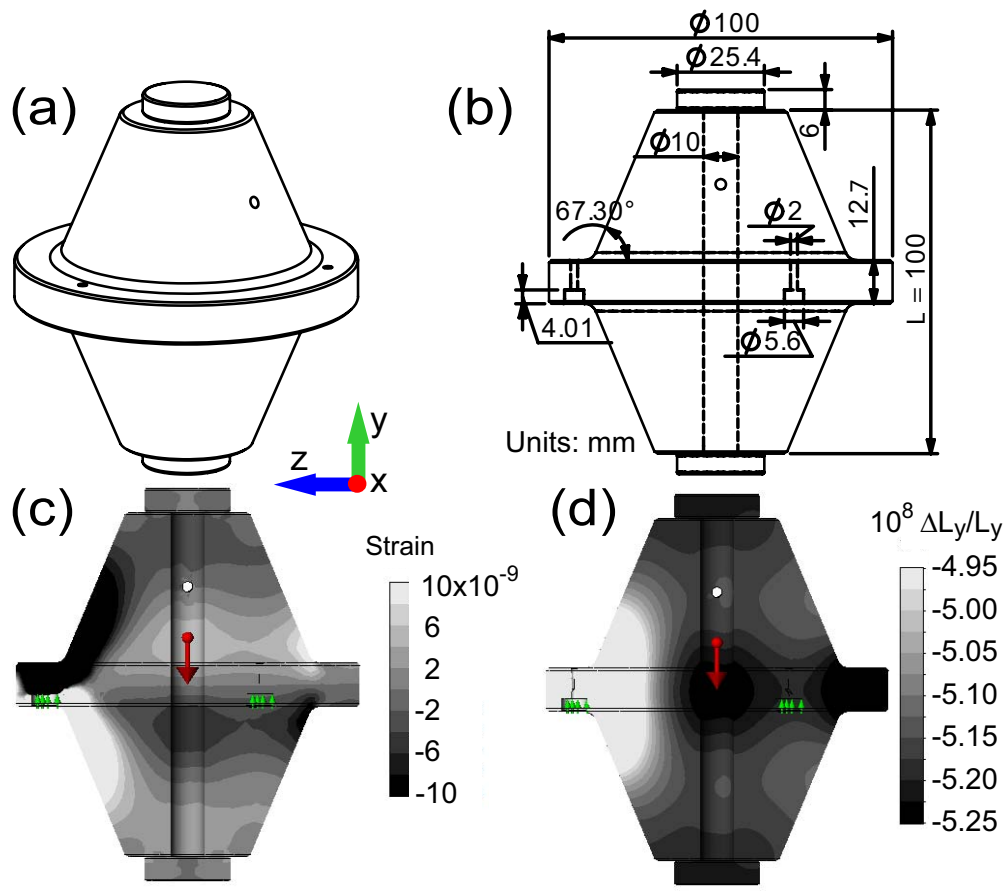

FIG. 7. (Color online) Tapered cavity horizontally mounted near its midplane. The optical axis of the cavity is along the $y$ axis. Three sets of mounting holes are drilled in the middle flange. (a) Three-dimensional view of the cavity (b) Dimension of the cavity. (c) and (d) are strain and displacement distributions along the optical axis in an axial cross section, respectively. The strain distribution is antisymmetric with respect to the midplane. The cavity length is insensitive to the vertical acceleration because the two mirrors move downward roughly in an equal amount.

fect, we numerically model a tapered cavity vertically supported near its midplane. This numerical modeling also provides valuable guidance for the optimization of the supporting position. Additionally, we examine the mirror displacement due to horizontal accelerations in the vertical mounting method.

\section{Tapered cavity}

Materials around the rims of a cylindrical spacer contribute less to the structural stiffness, and sometimes they are removed in the process of machining. Figure 7(a) shows such a tapered cavity with a flange at the middle for vertical support. As shown in Fig. 7(b), three sets of mounting holes are drilled in the flange and they are evenly distributed on a circumference. Each set of these mounting holes consists of two concentric holes with different diameters. The upper mounting holes (those with a smaller diameter) add the flexibility of vertical suspension with thin wires, but are not imperative for the current analysis. The horizontal surfaces [Fig. 7(b)] inside the holes are used as supporting surfaces and are vertically constrained in the calculation.

Figures 7(c) and 7(d) display the strain and displacement of the cavity along the vertical direction in an axial cross section, respectively. The compression and stretch of the material are illustrated by the nearly antisymmetric distribution of strain [Fig. $7(\mathrm{c})]$ with respect to the midplane. Similarly, the symmetric distribution of displacement [Fig. 7(d)] shows that two mirrors move downward roughly an in qual amount. To inspect this effect in detail, we plot the mirror displacements in Fig. 8. Figure 8(a) shows the displacements of the two mirrors with three supporting surfaces located exactly in the midplane. Obviously, both mirrors move downward and sag in the middle. The differential displacement in the central region of the mirror is $\sim 0.007 \mathrm{~nm}$, corresponding to a fractional length change of $\sim 7 \times 10^{-11} / \mathrm{g}(\sim 40 \mathrm{kHz} / \mathrm{g}$ for optical frequency change at $532 \mathrm{~nm}$ ).
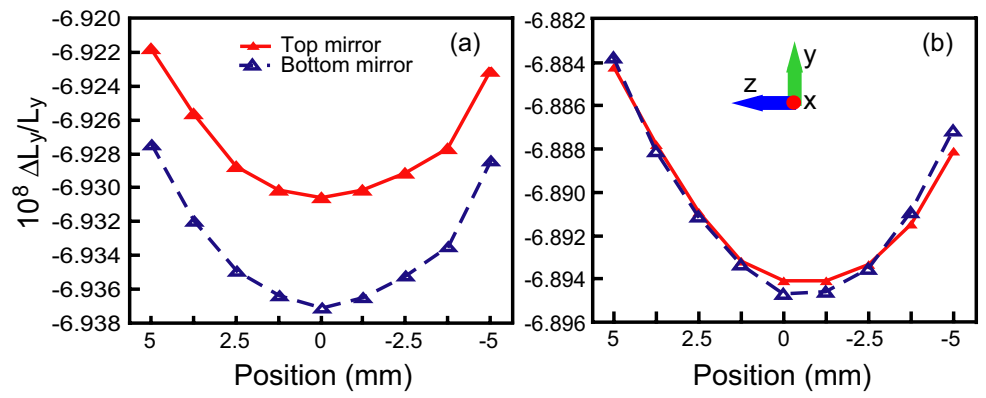

FIG. 8. (Color online) Mirror displacements for a tapered cavity vertically mounted. The horizontal scale represents the transverse position (along the $z$ axis) on the reflecting surface of the mirror, with the origin denoting the center of the mirror. (a) The cavity is supported at the midplane. Both mirrors move downward and sag in the middle. The mounting holes on the flange add structural weakness to the lower half of the cavity spacer, resulting in a residual length change of $\sim 0.007 \mathrm{~nm}\left(\sim 7 \times 10^{-11} / \mathrm{g}\right.$, fractional or $\sim 40 \mathrm{kHz} / \mathrm{g}$, optical frequency change at $532 \mathrm{~nm}$ ). (b) Supporting surfaces moved downward $(2.3 \mathrm{~mm})$ to compensate for the structural weakness in the lower half of the cavity flange, resulting in nearly identical movements of the two mirrors and a zero length change. 

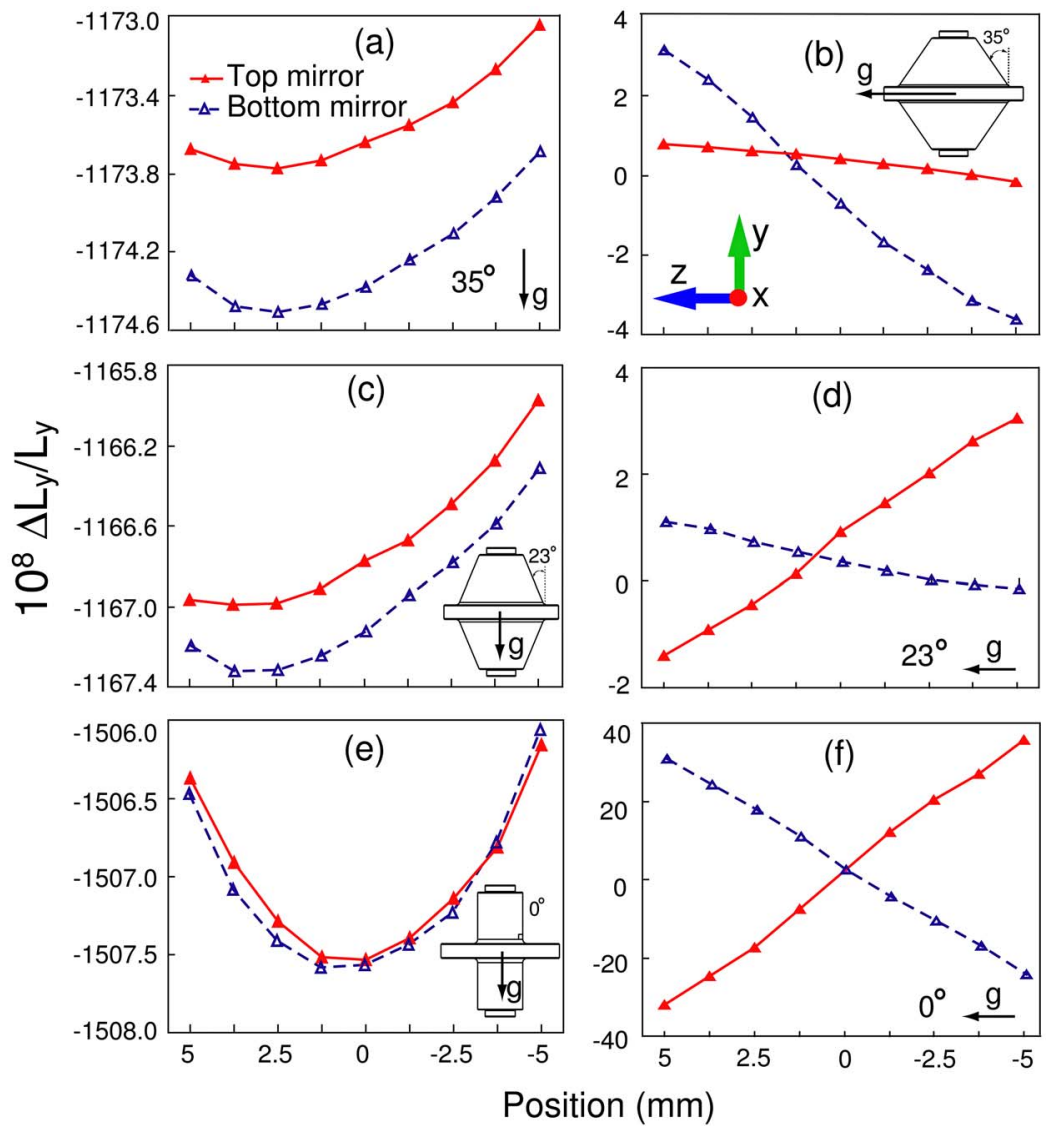

FIG. 9. (Color online) Mirror displacements of cavities with different taper angles. The horizontal scale represents the transverse position (along the $z$ axis) on the reflecting surface of the mirror, with the origin denoting the center of the mirror. Cavities are supported in the midplane. Taper angles and accelerations are indicated in the figure. Left panels (a), (c), and (e): vertical acceleration. Right panels (b), (d), and (f): horizontal acceleration pointing to the left. Each row of two panels is for a certain taper angle. Vertical acceleration induces relatively large residual length change in cavities with large taper angles. For horizontal acceleration, the tilting angles of two mirrors in cylindrical cavity (f) are nearly one order of magnitude larger than those of tapered cavities [(b) and (d)].
The residual length change shown in Fig. 8(a) can be further reduced. As shown in Fig. 7(b), weight-bearing surfaces are formed by drilling holes in the flange, which induces extra deformations in those regions around the mounting holes. Accordingly, the supporting surfaces can be moved downward to compensate for the weakened structure near the support. Indeed, a full compensation can be achieved when the surfaces are lowered $2.3 \mathrm{~mm}$ away from the midplane. Figure 8(b) shows the mirror displacement with optimized supporting surfaces. Alternatively, reducing the hole size can achieve the same result, but will tighten the position tolerance of the holes on the flange.

\section{Tapered cavity with different angles}

With the high resolution provided by FEA, various design factors can be numerically modeled prior to the experiments. As an example, here we show the numerical analysis for cavities with different taper angles. Three taper angles are chosen and the cavities are supported at the midplane. The supporting surfaces are fixed only in the vertical direction when the acceleration is applied downward, but are constrained in all directions when the acceleration is in the horizontal. For comparison, the areas of the two end surfaces and the outer diameter of the flange are fixed while the taper angle is varied. Figure 9 summarizes the numerical results. Each row in the figure plots mirror displacements for a certain taper angle (indicated in the figure). In each row, left and right panels show displacements under vertical and horizontal accelerations, respectively. In the case of vertical accel- eration [Figs. 9(a), 9(c), and 9(e)], cavities with large taper angles exhibit relatively large residual changes in optical length. Note that in Figs. 9(a) and 9(c), regions with the largest vertical sag are shifted away from the central region of the mirrors, though the three supporting holes are symmetrically located on the flange. As the acceleration is switched to the horizontal direction, pointing toward the left, two mirrors are tilted due to the bending of the spacer. For the cylindrical cavity [Fig. 9(f)], the tilting angles of two mirrors are nearly one order of magnitude larger than those of tapered cavities [Figs. 9(b) and 9(d)]. Also note that in Figs. 9(b) and 9(d), two mirrors exhibit unequal tilting angles because of the structural weakness induced by the holes on the lower half of the flange.

\section{Improved horizontal mounting schemes}

Thus far we have examined cavities mounted either horizontally or vertically. The distance between two mirrors in cavities mounted vertically can be made considerably insensitive to vertical vibrations. For cavities horizontally supported from the bottom with strong restrictions on the bending of the cavity spacer, vertical compression is unavoidably accompanied by horizontal expansion due to the nonzero Poisson ratio. Consequently, the similar vibration insensitivity realized in the vertical cavities is generally not seen in their horizontal counterparts. Nevertheless, horizontal cavities with vibration immunity are strongly favored in several important experiments. One example is the test of Lorentz invariance [Michelson-Morley (MM) experiment] in which 


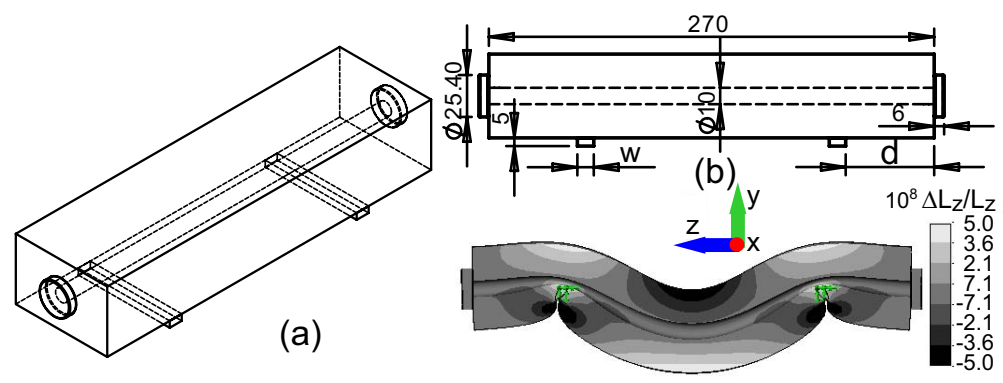

(c)

FIG. 10. (Color online) Cavity made from a rectangular bar and supported by two beams. The cavity has a cross section of $76.2 \mathrm{~mm}$ $\times 50.8 \mathrm{~mm}$ and a length of $270 \mathrm{~mm}$, which is different from other $100-\mathrm{mm}$ cavities discussed in this paper. $d$ and $w$ are varied in the numerical analysis to inspect the mirror tilt and displacement along the optical $(z)$ axis at various mounting positions. (a) Three-dimensional view of the cavity and its support. (b) Dimension of the cavity. (c) Cavity deformation and the distribution of the strain along the $z$ axis in an axial cross section. Note that the deformation has been amplified by a factor of $5 \times 10^{6}$.

the optical resonance frequencies of two orthogonal cavities are alternately probed [40-42]. The remainder of this section scrutinizes various supporting positions that can lead to improved vibration insensitivity in the horizontal cavities.

\section{Rectangular-bar cavity supported underneath}

Bending of the cavity spacer can affect the distance between the two mirrors. Here we explore this degree of freedom, using a cavity made from a rectangular bar and supported from the bottom by two beams. Figure 10 gives the dimensions of the cavity and the supporting beams. In our numerical modeling the height of the beam is fixed at $5 \mathrm{~mm}$ while two parameters are adjusted: namely, the location $d$ and the width $w$ of the beam.

Figure 11 plots the mirror displacements for six combinations of $d$ and $w$ (values indicated in the figure), which cover three typical widths of the supporting beam. At each $w$, three results with increasing values of $d$ are plotted and arranged in the figure as a row of three panels. For a given $w$, we find the Airy point at which the parallelism of the two mirrors is obtained (middle panel of each row in Fig. 11). Interestingly, with a specific combination of $d$ and $w$, the cavity length responds insensitively to the vertical accelerations while the two mirrors remain parallel to each other, as shown in Fig. 11(e). When $w$ changes from $3 \mathrm{~mm}$ to $10 \mathrm{~mm}$, the differential displacement between the two mirrors reverses sign. The same sign reversal also happens when $w$ is fixed at $4.41 \mathrm{~mm}$ and the supporting beams move from one side of the Airy point to the other.

We attribute these phenomena to the combined effect of gravity-induced vertical compression and bending of the cavity spacer. The weight load between two supporting beams bends the middle of the spacer downward, inducing stress components along the optical axis of the cavity. These stress components point to the center of the cavity and hence tend to decrease the cavity length. The counteracting stress components are also present in the cavity spacer because of the warp of the two cavity ends and because of the gravityinduced vertical compression. With a combination of $w$ $=4.41 \mathrm{~mm}$ and $d=53.62 \mathrm{~mm}$, various length-changing effects are exactly balanced out and the cavity length is insensitive to vertical accelerations, as shown in Fig. 11(e) [43]. Using this favored geometry we remeasure the displacement

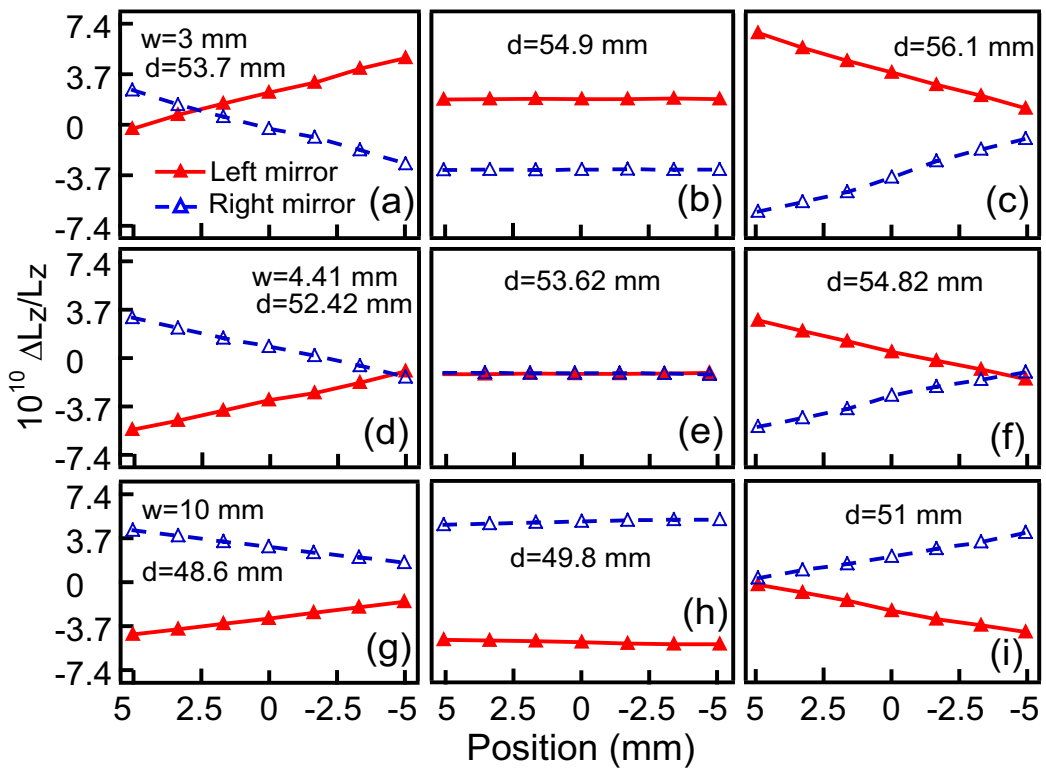

FIG. 11. (Color online) Mirror displacements of a cavity made from a rectangular bar and supported by two beams with different widths and positions ( $w$ and $d$ indicated in the figure). The horizontal scale represents the transverse position (along the $y$ axis) on the reflecting surface of the mirror, with the origin denoting the center of the mirror. See Fig. 10 for coordinate system. (a)-(c) $w=3 \mathrm{~mm}$. (d)-(f) $w=4.41 \mathrm{~mm}$. (g)-(i) $w=10 \mathrm{~mm}$. For a given beam width $w$, the Airy point can be found where the parallelism of the two mirrors is obtained [(b), (e), and (h)]. When $w=4.41 \mathrm{~mm}$ and $d=53.62 \mathrm{~mm}$ (e), the cavity length responds insensitively to the vertical accelerations while the two mirrors remain parallel to each other. 


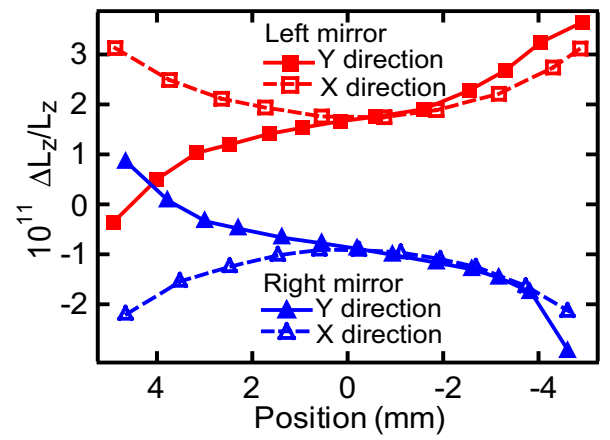

FIG. 12. (Color online) Mirror displacements of a horizontal cavity (Fig. 10) with optimized structural support $[w$ and $d$ indicated in Figs. 11(d) and 11(e)]. The displacements are measured along the $x$ and $y$ axes on the reflecting surface of the mirror (see Fig. 10 for coordinate system). The origin of the horizontal scale denotes the center of the mirror.

of the mirror along two orthogonal axes and plot the result in Fig. 12 with enlarged vertical scale [cf. Fig 11(e)]. Obviously, the vibration sensitivity can be further reduced by iterative adjustments of $w$ and $d$. When $w$ is fixed at $4.41 \mathrm{~mm}$ and $d$ is changed from $52.42 \mathrm{~mm}$ to $54.82 \mathrm{~mm}$ [Figs. 11(d) and 11(f), respectively], the weight load between the two beams decreases, resulting in diminished length-contraction stress components and hence a net increase of the cavity length in addition to the mirror tilt. This effect is clearly demonstrated by the mirror displacements shown in Figs. 11(d) and 11(f).

The change in cavity length $\Delta L$ is also inspected in a broad range of the width $w$. At each $w$ we first adjust $d$ to ensure the parallelism of the two mirrors and then obtain $\Delta L$ by taking the differential displacement of the two mirrors. Figure 13 shows the trend of $\Delta L$ as $w$ is varied from $3 \mathrm{~mm}$ to $135 \mathrm{~mm}$. The rightmost data point $(w=135 \mathrm{~mm})$ represents the situation in which the bottom of the cavity is fully supported by a flat plate. In this case the cavity is elongated because of the gravity-induced vertical compression.

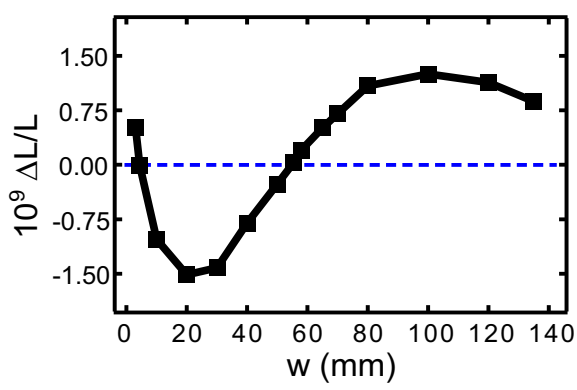

FIG. 13. (Color online) Change in cavity length as a function of the width of the supporting beam. The spacer of the cavity is a rectangular bar. The cavity is supported by two beams (see Fig. 10). The beam height is fixed at $5 \mathrm{~mm}$ in all calculations. $\Delta L$ at each $w$ is obtained with cavity supported at corresponding Airy point. At two widths $(w=4.4$ and $55.4 \mathrm{~mm}) \Delta L$ is insensitive to vertical accelerations. The cavity is shortened in the range of $4.4 \mathrm{~mm}<w$ $<55.4 \mathrm{~mm}$, but is elongated outside this range. The rightmost data point $(w=135 \mathrm{~mm})$ represents the situation in which the bottom area of the cavity is fully supported by a flat plate.

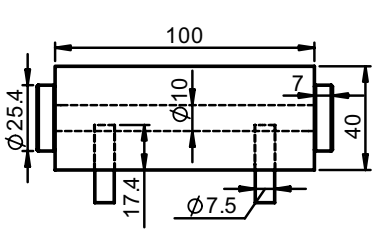

(a)

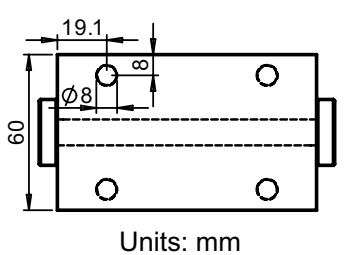

(b)

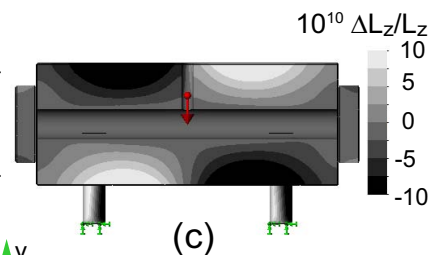

(c)

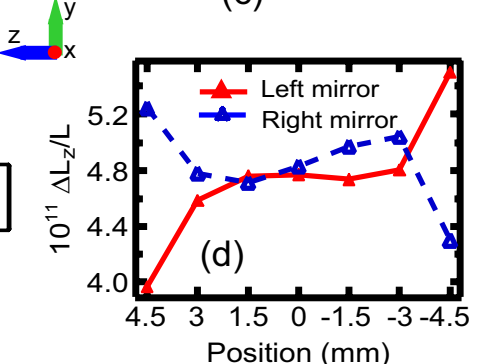

FIG. 14. (Color online) Cavity made from a rectangular bar and supported by four posts. Supporting cavity at different heights is made possible by drilling four blind holes on the bottom of the spacer. In the numerical modeling the location and the depth of the supporting holes are varied to lower the vibration sensitivity and to ensure the parallelism of the two mirrors. (a), (b) Relevant dimensions. (c) Distribution of the displacement in an axial cross section. (d) Mirror displacements along the $z$ axis after the optimization of the depth and the location of the mounting holes. The horizontal scale represents the transverse position (along the $y$ axis) on the reflecting surface of the mirror, with the origin denoting the center of the mirror.

When $w$ is within (outside) the range of $4.4 \mathrm{~mm}<w$ $<55.4 \mathrm{~mm}$, the cavity is shortened (elongated). Note that, for the cavity geometry and the beam height we choose, there are two widths $(w=4.4$ and $55.4 \mathrm{~mm})$ at which the cavity length is insensitive to vertical accelerations.

Unfortunately, such a favorable combination of the width and location of the supporting beams is not ubiquitous in cavities made from rectangular bars with different dimensions. For example, numerical calculation shows that optimized parameters do not exist for a similar cavity with a length of $100 \mathrm{~mm}$. Compared with the 270-mm cavity, this shortened cavity has an increased stiffness. As a result, the limited bending of the cavity spacer cannot fully compensate the horizontal extension resulting from gravity-induced vertical compression.

\section{Cavity with supporting height optimized}

As illustrated in Sec. III A 1, a horizontal cavity resting on its bottom is elongated because of vertical compression, whereas the same cavity suspended from the top contracts in the horizontal direction. Supporting the cavity at a height in between these two extremes can potentially suppress the change of the optical length induced by vertical accelerations. We investigate this possibility by using a rectangularbar cavity supported by four posts.

Figures 14(a) and 14(b) show the cavity with relevant dimensions. Supporting the cavity at different heights is made possible by drilling four blind holes on the bottom of the cavity spacer. In the numerical modeling, we first find the Airy point such that the two mirrors are always parallel to 


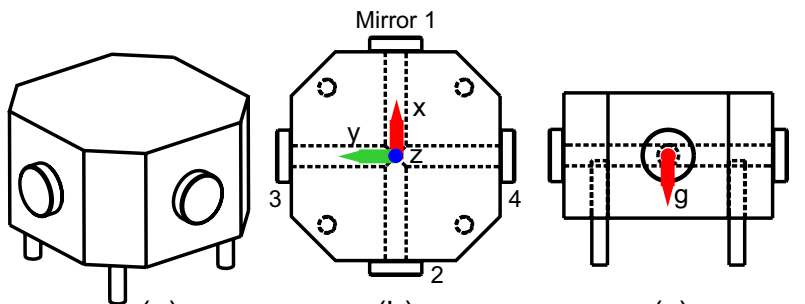

(a)

(b)
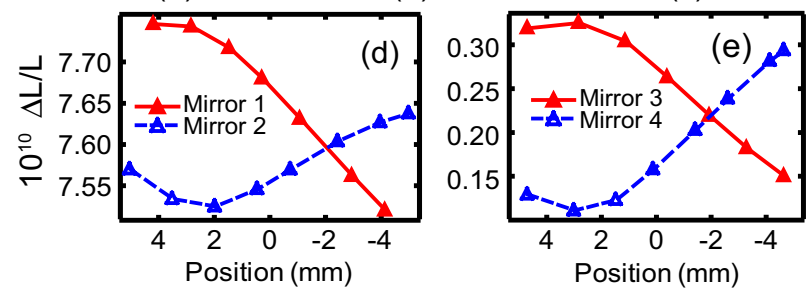

FIG. 15. (Color online) A compound cavity designed for the test of Lorentz invariance. (a) Three-dimensional view of the cavity and its support. (b) Top view. (c) Side view. Two holes perpendicular to each other are bored through the cavity spacer. High-reflection mirrors are attached to the end surfaces, forming two orthogonal cavities. Vibration insensitivity along the $x$ and $y$ axes is obtained by adjusting the depth and the position of the four mounting holes. (d) and (e) show mirror displacements, respectively, along the $x$ and $y$ axes after the optimization. The horizontal scale represents the transverse position (along the $z$ axis) on the reflecting surface of the mirror, with the origin denoting the center of the mirror.

each other despite the vertical accelerations. However, a residual change in optical length is usually present after this adjustment. We subsequently vary the depth of the holes to reduce this length residual. The process is iterated until both the parallelism of the mirrors and the cancellation of the mirror displacements are simultaneously obtained. Figure 14(d) gives the mirror displacements after the optimization of the location and the depth of the mounting holes.

\section{Cavity design for testing Lorentz invariance}

Based on the analysis of the horizontally mounted linear cavities, we introduce a cavity design for the test of Lorentz invariance in the photon sector of the standard model extension (SME) $[44,45]$. In electrodynamics the test has been performed with modern versions of the Michelson-Morley experiments using room-temperature optical cavities [40], cryogenic sapphire optical resonators [41,42], and cryogenic microwave oscillators $[46,47]$. In a typical experiment the resonant frequencies of two orthogonal cavities are continuously compared as they either move along with the rotation of the Earth or are actively rotated on a laboratory horizontal platform.

The horizontal mounting scheme introduced in the preceding section can be simultaneously applied to two orthogonal directions. Figure 15 shows a cavity designed for this purpose. The spacer has through holes for two optical axes that are perpendicular to each other. Four high-reflection mirrors are attached to the side faces of the spacer, forming two orthogonal cavities in the horizontal plane. For a given diameter of the supporting holes, the depth of the holes and their horizontal positions are varied until an optimization in two orthogonal directions is obtained. Figures 15(d) and 15(e) show respectively the mirror displacements along the $x$ and $y$ axes after the optimization [48]. Clearly, vibration resistance in both directions is attainable. In fact, a third cavity in the $z$ axis can also be added and the vibration resistance can be achieved in all three directions with the aid of another degree of freedom - the diameter of the holes.

Several potential advantages are associated with this compounded cavity. The vibration immunity of the cavity will in general reduce the high-frequency noise superposed on the signal obtained from long-term measurements based on the Earth's rotation. Moreover, the compact system introduced here is well suited for active-rotation experiments. These experiments operate at relatively high modulation frequencies compared with the Earth's rotational and orbital frequencies, thereby taking advantage of the short-term stability of optical local oscillators $(10-100 \mathrm{~s})$. In active-rotation mode, it is possible that the rotation frequency can be comparable with low Fourier frequencies $(\leqslant 1 \mathrm{~Hz})$ in the vibration spectrum. These low-frequency components find their way more easily to the cavity and develop noise sidebands close enough to the carrier to be picked up by the detection bandwidth. The vibration immunity of the cavity will help to suppress this seismic interference and other spurious signals arising from the periodical perturbations from the rotation. In addition, the double-cavity-in-one-piece configuration can possibly offer improved common-mode rejection of the thermal drift and isotropic material creep [49]. These preferred features, if realized, are crucial for long-term measurements that search for Lorentz-violating signals modulated by the Earth's rotational and orbital movements.

However, for this design to be implemented in activerotation mode, special cautions must be exercised. Jitters of centrifugal acceleration can induce unequal stretches of the two cavities, which in turn modulate the frequency difference. This perturbation can possibly be alleviated by centering the cavity on the rotation stage and by using a narrow detection bandwidth at twice the angular frequency. An active control of the rotational speed may also be used to level off the centrifugal stretch. The tilt of the rotation stage can also modulate the frequency difference, contaminating the signal at twice the rotation frequency [46]. In addition to the leveling of the rotation stage, one can further optimize the geometry of the compounded cavity and its support to reduce the vibration sensitivity both in the vertical and horizontal planes.

\section{ACCURACY OF NUMERICAL ANALYSIS}

Because extremely small displacements are involved in the numerical modeling of cavities, designs based on these analyses must be carefully checked prior to their experimental implementation. The error can arise from the calculation itself or may be caused by limited knowledge of the initial conditions such as the material properties and the contact between the cavity and the structural support. In this section we consider, from a computational perspective, major factors that can potentially affect the accuracy of the numerical modeling. 

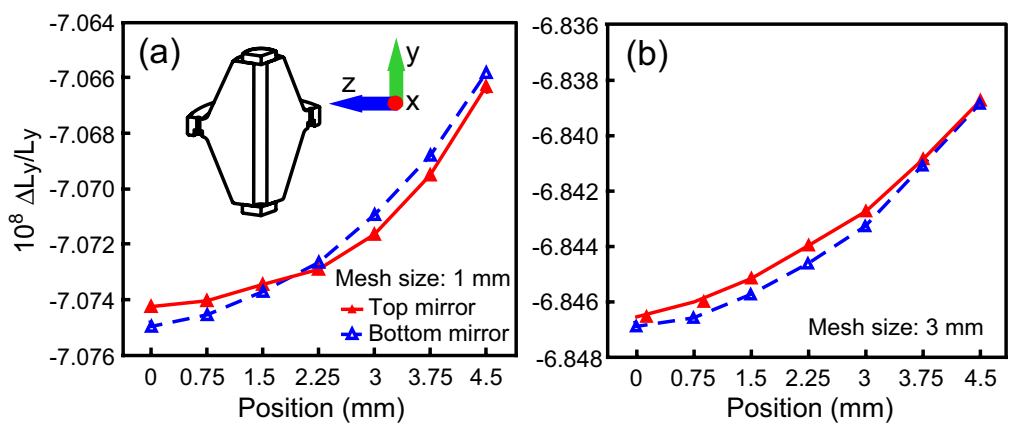

FIG. 16. (Color online) Numerical analysis of a tapered cavity with two different mesh sizes. The horizontal scale represents the transverse position on the reflecting surface of the mirror, with the origin denoting the center of the mirror. Only one third of the cavity is used in the FEA to take advantage of geometrical symmetry. (a) Mesh size: $1 \mathrm{~mm}$. (b) Mesh size: $3 \mathrm{~mm}$. The displacements of two mirrors are different for the two mesh sizes. But the differential displacements obtained with two mesh sizes are nearly the same.

\section{A. Mesh size}

Mesh size is one of the major parameters in FEA that affect the accuracy of the numerical modeling, especially in dealing with extremely small displacements $\left(\sim 10^{-11}\right.$, fractional). A small mesh size is preferred in order to obtain the required resolution and accuracy, but reductions in mesh size are limited by computing resources and often lead to an excessive amount of computing time. To evaluate the potential errors introduced by using finite mesh sizes, we perform calculations for the same cavity but with different mesh sizes. As an example, Fig. 16 plots mirror displacements for a tapered cavity with two different mesh sizes (1 and $3 \mathrm{~mm})$. To take advantage of the geometrical symmetry, only one-third of the cavity is modeled (see the inset of Fig. 16). The two results in Fig. 16 indicate that the magnitude of the displacement of each mirror can change for different mesh sizes but the differential displacement is nearly unaffected by the mesh size. Additional calculations on various cavities confirmed this observation.

\section{B. Constraints of supporting surfaces}

In the static analysis the structural support is constrained to prevent the translational and rotational movements of the mechanical structure. As an example, consider the tapered cavity introduced in Sec. III B 1. The supporting surfaces can be fixed in all three directions, or they can move freely in the horizontal plane. In the numerical modeling, these two different constraints lead to a fractional change of $2 \times 10^{-10}$ in the optical length between the two mirrors. Restriction only in the vertical direction is more close to the practical situation where the cavity rests on three posts but is not bonded to them. Nonetheless, this vertical restraint is only an ideal case that cannot fully describe the real contact between the cavity and the supporting surface.

We perform two additional diagnostic calculations to identify potential errors that can arise from the contact problem. The first one models the tapered cavity supported by three aluminum posts whose end surfaces are bonded to the cavity. Subsequent calculation is performed with a soft layer (indium) inserted between the cavity and each aluminum post. In the second configuration, the shear deformation of the soft layer partially simulates the sliding between two contacting surfaces. A comparison between these two calculations helps to resolve the ambiguity arising from the sliding between the cavity and the aluminum posts in a real contact problem. Figure 17 shows the mirror displacements with and without the soft layers between the contacting surfaces. With the indium layers added, the mirror displacement increases by $4 \times 10^{-9} / \mathrm{g}$ (fractional), but the differential displacement varies by only $\sim 2 \times 10^{-11} / \mathrm{g}$ (fractional), suggesting a negli-

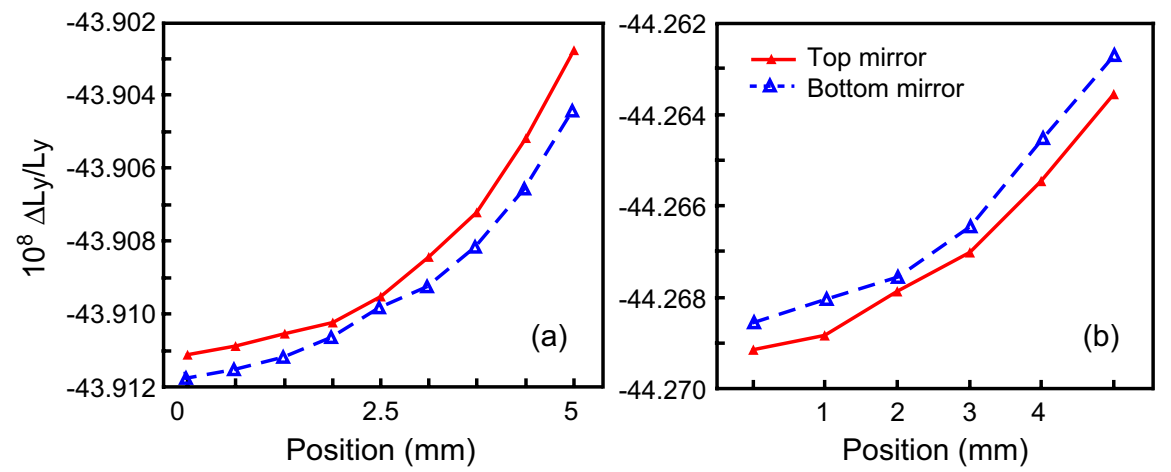

FIG. 17. (Color online) Mirror displacements of a tapered cavity vertically supported by three aluminum posts. Only one third of the cavity is used in the FEA to take advantage of the geometrical symmetry. (a) Cavity supported by three aluminum posts. (b) Indium layers $(1 \mathrm{~mm}$ thick) are inserted between the cavity and the aluminum posts. When indium layers are added the mirror displacement increases by $4 \times 10^{-9} / \mathrm{g}$ (fractional), but the differential displacement varies by only $\sim 2 \times 10^{-11} / \mathrm{g}$ (fractional). 
gible effect of the sliding between two contacting surfaces. In the future, FEA packages with sophisticated tools for investigating contact problems will lead to an improved accuracy and confidence in the cavity design.

\section{Material properties and machining tolerance}

Material properties of cavities can possibly deviate from their nominal values. For a cavity design intended for vibration immunity, a hypersensitive dependence on the material properties will render the experimental implementation impossible. To examine the stability of the numerical modeling, we repeat the calculations with deliberately varied material properties such as the elastic modulus, the Poisson ratio, and the mass density. These parameters are typically varied by $5 \%$ in the calculation, and we find that a vibration sensitivity below $\sim 7 \times 10^{-11} / \mathrm{g}$ can still be achieved. Consequently, optimized supporting positions for vibration resistance are attainable despite a modest spread of the material properties that can arise from the fabrication process or from fluctuations of the ambient temperature.

Various dimensional changes can potentially undermine the optimized cavity design. We perform a series of calculations using modified cavity dimensions to account for the typical manufacturing tolerance. For the tapered cavity introduced in Sec. III B, its vibration sensitivity is below 1 $\times 10^{-11} / \mathrm{g}$ at the nominal length. We shorten the top part of the cavity by $0.5 \mathrm{~mm}$, and this dimensional modification results in a vibration sensitivity of $6 \times 10^{-11} / \mathrm{g}$, which is still about 30 times smaller than that of a horizontal cavity supported from the bottom. The allowed tolerances for other crucial dimensions are also determined in a similar manner for a given design goal. In addition, the optimized location and the depth of the mounting holes obtained for the tapered cavity are also verified against position errors that can potentially arise from machining of cavities.

\section{SUMMARY AND CONCLUSION}

FEA is used to quantitatively analyze the elastic deformation of Fabry-Perot cavities with various shapes and mounting configurations. At low vibration frequencies, a static analysis of cavity deformations can be adopted to simplify the numerical modeling. Vibration-induced fluctuations in cavity length are closely examined for several experimental configurations, which include a horizontally mounted cylindrical cavity, a tapered cavity vertically supported near its midplane, and two horizontal cavities with modified mounting configurations. By vertically supporting the cavity near its midplane, the influence of vibration on the cavity length can be reduced. Similar vibration resistance can also be realized in horizontal cavities by adjusting the related structural supports. This latter observation facilitates a cavity design for the test of Lorentz invariance. The accuracy and feasibility of experimental realization of these mounting schemes have also been discussed.

Considering the diversity of the geometry, installation, and application of Fabry-Perot cavities, it is impossible to cover them all in a single article here. Fortunately, some general observations can still be distilled. Both vertically and horizontally mounted cavities can be optimized such that their critical dimensions can be protected from vibrational perturbations through a careful design of the geometry and the related structural support. The vibration sensitivity of vertical cavities can be reduced by two orders of magnitude with controlled machining tolerance $(0.1-0.2 \mathrm{~mm})$. Optimized horizontal cavities exhibit similar vibration immunity, but may demand a tighter machining and mounting tolerance. Horizontal vibrations induce nearly symmetrical mirror tilt without additional cavity elongation in vertical cavities, but their influence on the horizontal cavities should be examined in detail for each specific design. For both vertical and horizontal configurations, further improvements are possible by imposing tighter tolerances on relevant dimensions and by experimentally fine-tuning the mass distribution [6].

Because of the reduced vibration sensitivity via proper design of the cavity geometry and mounting scheme, one can choose a cavity length that minimizes other noise contributions such as from the thermal noise and birefringent noise [50] of the mirror coating. This is especially important for cavity design under the constraints of currently available cavity materials and mirror coatings. The horizontal and vertical mounting schemes discussed in this paper can be adopted, perhaps with necessary modifications, by various systems such as cryogenic optical resonators and those intended for space-based experiments.

\section{ACKNOWLEDGMENTS}

We wish to thank M. Notcutt and L.-S. Ma for stimulating discussions. Suggestions from J. Li at NIM on the issues of precision optical fabrication are gratefully acknowledged by L.C. This research is in part supported by the National Key Basic Research Program (No. 2005CB724501) of China. Work at JILA is supported by NASA, NIST, ONR, and NSF. J.Y. is also a member of the Quantum Physics Division of NIST.
[1] R. W. P. Drever, J. L. Hall, F. V. Kowalski, J. Hough, G. M. Ford, A. J. Munley, and H. Ward, Appl. Phys. B: Photophys. Laser Chem. 31, 97 (1983).

[2] C. Salomon, D. Hils, and J. L. Hall, J. Opt. Soc. Am. B 5, 1576 (1988).

[3] M. Zhu and J. L. Hall, J. Opt. Soc. Am. B 10, 802 (1993).

[4] B. C. Young, F. C. Cruz, W. M. Itano, and J. C. Bergquist,
Phys. Rev. Lett. 82, 3799 (1999).

[5] S. A. Webster, M. Oxborrow, and P. Gill, Opt. Lett. 29, 1497 (2004).

[6] M. Notcutt, L.-S. Ma, J. Ye, and J. L. Hall, Opt. Lett. 30, 1815 (2005).

[7] H. Stoehr, F. Mensing, J. Helmcke, and U. Sterr, Opt. Lett. 31, 736 (2006). 
[8] L. S. Ma, J. Ye, P. Dubé, and J. L. Hall, J. Opt. Soc. Am. B 16, 2255 (1999).

[9] J. Ye, L. S. Ma, and J. L. Hall, Opt. Lett. 21, 1000 (1996).

[10] J. Ye, L. S. Ma, and J. L. Hall, J. Opt. Soc. Am. B 15, 6 (1998).

[11] R. J. Rafac, B. C. Young, J. A. Beall, W. M. Itano, D. J. Wineland, and J. C. Bergquist, Phys. Rev. Lett. 85, 2462 (2000).

[12] J. Stenger, C. Tamm, N. Haverkamp, S. Weyers, and H. R. Telle, Opt. Lett. 26, 1589 (2001).

[13] P. Dubé, L. Marmet, A. A. Madej, and J. E. Bernard, in 2004 Conference on Precision Electromagnetic Measurements Digest, edited by G. Jones (IEEE, Piscataway, NJ, 2004), p. 283.

[14] H. S. Margolis, G. P. Barwood, G. Huang, H. A. Klein, S. N. Lea, K. Szymaniec, and P. Gill, Science 306, 1355 (2004).

[15] A. D. Ludlow, M. M. Boyd, T. Zelevinsky, S. M. Foreman, S. Blatt, M. Notcutt, T. Ido, and J. Ye, Phys. Rev. Lett. 96, 033003 (2006).

[16] D. J. Wineland, J. C. Bergquist, W. M. Itano, F. Diedrich, and C. S. Weimer, in The Hydrogen Atom, edited by G. F. Bassani, M. Inguscio, and T. W. Hänsch (Springer-Verlag, Berlin, 1989).

[17] H. Katori, M. Takamoto, V. G. Pal'chikov, and V. D. Ovsiannikov, Phys. Rev. Lett. 91, 173005 (2003).

[18] S. A. Diddams et al., Science 293, 825 (2001).

[19] M. Takamoto, F.-L. Hong, R. Higashi, and H. Katori, Nature (London) 435, 321 (2005).

[20] Z. W. Barber, C. W. Hoyt, C. W. Oates, L. Hollberg, A. V. Taichenachev, and V. I. Yudin, Phys. Rev. Lett. 96, 083002 (2006).

[21] R. H. Dicke, Phys. Rev. 89, 472 (1953).

[22] T. Udem, J. Reichert, R. Holzwarth, and T. W. Hänsch, Phys. Rev. Lett. 82, 3568 (1999).

[23] J. Reichert, R. Holzwarth, T. Udem, and T. W. Hänsch, Opt. Commun. 172, 59 (1999).

[24] H. R. Telle, G. Steinmeyer, A. E. Dunlop, J. Stenger, D. H. Sutter, and U. Keller, Appl. Phys. B: Lasers Opt. 69, 327 (1999).

[25] S. A. Diddams, D. J. Jones, J. Ye, S. T. Cundiff, J. L. Hall, J. K. Ranka, R. S. Windeler, R. Holwarth, T. Udem, and T. W. Hänsch, Phys. Rev. Lett. 84, 5102 (2000).

[26] S. T. Cundiff and J. Ye, Rev. Mod. Phys. 75, 325 (2003).

[27] S. A. Diddams, J. Ye, and L. Hollberg, in Femtosecond Optical Frequency Comb Technology: Principle, Operation, and Application, edited by J. Ye and S. T. Cundiff (Springer, New York, 2005)

[28] S. G. Porsev, A. Derevianko, and E. N. Fortson, Phys. Rev. A 69, 021403(R) (2004).

[29] R. Santra, E. Arimondo, T. Ido, C. H. Greene, and J. Ye, Phys. Rev. Lett. 94, 173002 (2005).

[30] A. V. Taichenachev, V. I. Yudin, C. W. Oates, C. W. Hoyt, Z. W. Barber, and L. Hollberg, Phys. Rev. Lett. 96, 083001 (2006).

[31] T. Hong, C. Cramer, E. Cook, W. Nagourney, and E. N. Fortson, Opt. Lett. 30, 2644 (2005).
[32] J. L. Hall, M. Notcutt, L.-S. Ma, and J. Ye (unpublished).

[33] A. Y. Nevsky, M. Eichenseer, J. von Zanthier, and H. Walther, Opt. Commun. 210, 91 (2002).

[34] M. Notcutt, C. T. Taylor, A. G. Mann, R. Gummer, and D. G. Blair, Cryogenics 36, 13 (1996).

[35] J. Bergquist (private communication).

[36] M. Zhu and J. L. Hall, in Atom, Molecular, and Optical Physics: Electromagnetic Radiation, edited by F. B. Dunning and R. G. Hulet (Academic Press, San Diego, 1997), Vol. 29C.

[37] J. L. Hall, M. Notcutt, and J. Ye, in Laser Spectroscopy XVII, edited by E. Hinds, A. Ferguson, and E. Riis (World Scientific, Singapore, 2006).

[38] K. Numata, A. Kemery, and J. Camp, Phys. Rev. Lett. 93, 250602 (2004).

[39] M. Notcutt, L.-S. Ma, A. D. Ludlow, S. M. Foreman, J. Ye, and J. L. Hall, Phys. Rev. A 73, 031804(R) (2006).

[40] A. Brillet and J. L. Hall, Phys. Rev. Lett. 42, 549 (1978).

[41] H. Muller, S. Herrmann, C. Braxmaier, S. Schiller, and A. Peters, Phys. Rev. Lett. 91, 020401 (2003).

[42] P. Antonini, M. Okhapkin, E. Goklu, and S. Schiller, Phys. Rev. A 71, 050101(R) (2005).

[43] The value of $d$ depends on the mesh size, especially for large mesh sizes ( $25 \mathrm{~mm}$ ). However, $d$ shows a convergent trend when the mesh size is reduced $(d=53.62 \mathrm{~mm}$ is obtained with $3 \mathrm{~mm}$ mesh size). We note that the calculation itself does not guarantee the accuracy of $d$ because of the approximation made about the contact between the cavity and its support. Rather, it is intended to reveal the trend of the length change when the position and dimension of the supporting beams are varied in a broad range. The accurate optimization of $d$ and $w$ relies on the experimental fine tuning.

[44] V. A. Kostelecký and M. Mewes, Phys. Rev. Lett. 87, 251304 (2001).

[45] V. A. Kostelecký and M. Mewes, Phys. Rev. D 66, 056005 (2002).

[46] P. L. Stanwix, M. E. Tobar, P. Wolf, M. Susli, C. R. Locke, E. N. Ivanov, J. Winterflood, and F. van Kann, Phys. Rev. Lett. 95, 040404 (2005).

[47] P. Wolf, S. Bize, A. Clairon, G. Santarelli, M. E. Tobar, and A. N. Luiten, Phys. Rev. D 70, 051902(R) (2004).

[48] Ideally, the two displacements plotted in Figs. 15(d) and 15(e) should be identical because of the geometrical symmetry involved. However, small differences in the $x$ and $y$ directions show up. In the numerical analysis the meshing of the cavity can have some local variations that are not perfectly symmetrical. This asymmetry can be removed by using a reduced mesh size. Nonetheless, a strict identity in the $x$ and $y$ directions cannot be achieved experimentally due to small but unavoidable dimensional deviations and the inhomogenous distribution of material properties inside the cavity.

[49] D. Hils and J. L. Hall, in Frequency Standards and Metrology, edited by A. D. Marchi (Springer-Verlag, Berlin, 1989).

[50] J. L. Hall, J. Ye, and L.-S. Ma, Phys. Rev. A 62, 013815 (2000). 